\title{
SKIN RESISTANCE CHANGES AND MEASUREMENTS OF PAIN THRESHOLD
}

\author{
By H. L. ANDREWS 1 \\ (United States Public Health Service, United States Public Health Service Hospital, \\ Lexington, Kentucky)
}

(Received for publication October 28, 1942)

When the end-point is reached in the HardyWolff method of measuring pain threshold, there is a sharp change in the subjective experience resulting from the stimulus. When the radiation intensity is below the level of the pain threshold, the sensation is one of heat alone. If the pain threshold is exceeded, there is a sensation of heat with an added factor which has been variously described as "a tweak," "a stab of pain," "a pin prick," or "a threat of tissue damage." The usefulness of this technique lies in the fact that however this endpoint is described, it can be duplicated on subsequent trials and after the administration of drugs.

The appreciation and description of this endpoint must involve a complex series of neural pathways, including the cerebral cortex. Skin resistance, on the other hand, is primarily under autonomic control, and is only secondarily affected by the cerebrum. Since the stimulus intensity can be adjusted to obtain equal subjective experiences, simultaneous measurements of pain threshold and skin resistance should furnish a means for differentiating between autonomic effects and those involving higher centers. These measurements might be expected to furnish an objective check on the accuracy of the subjective reports, for when the stimulus exceeds the pain threshold, there is a sharp increase in the emotional content of the stimulation and a sudden increase in the magnitude of the skin resistance change would be anticipated. The results obtained from such simultaneous measurements are described in the present report.

\section{METHODS}

Pain threshold measurements were made with apparatus essentially the same as that described by Hardy and Wolff (1). A 3-second stimulus was given at 30second intervals. The lamp supplying the stimulus was operated from A.C. through a voltage-regulating transformer and controlled by a Variac. Each stimulus in-

1 Present address: National Institute of Health, Bethesda, Maryland. tensity was measured by a wattmeter connected in the lamp circuit.

Skin resistance measurements were made from silver chloride electrodes, attached to the palm and dorsum of the hand with collodion. Contact to the skin was made through an isotonic electrode paste. In applying these electrodes, care was taken to avoid injuring the palmar skin, but the dorsum was rubbed vigorously with electrode paste to obtain a low resistance contact. The hand formed one arm of a Wheatstone bridge which was fed from a 1.5 volt cell through a potentiometer. This was adjusted to keep the electrode current below 5 microamperes, thus reducing disturbances due to polarization.

The output of the bridge was fed into a 2-stage, direct coupled amplifier which drove a Westinghouse type PA galvanometer. Photographic recording was on bromide paper. Auxiliary circuits were provided to record the incidence of the stimuli and the subjective reports.

Current was allowed to pass through the electrodes only during the actual measurements, which usually required about 3 or 4 minutes. When the effects of drugs were being studied, a run was made every 15 minutes after drug administration and when the effects lasted more than 2 or 3 hours, new electrodes were applied to eliminate the effects of cumulative polarization.

The pain threshold data were treated in the usual way, the increase over the pre-injection threshold being expressed as a percentage of this threshold and plotted against the time after the injection. The area under each skin resistance change was measured for a 20 -second interval with a planimeter. By dividing this area by the base line, the average height of the curve was obtained, and from the bridge calibrations, this was converted to ohms change and to percentage change. Although measurements of maximum change gave results essentially similar to those obtained from the average values, the latter were used in all calculations, since these represent more nearly the total integrated effect of the stimulus.

\section{RESULTS}

The study was carried out on 2 non-addicts and on a group of men who had previously been addicted to opiates but whose addiction had terminated at least 6 months prior to the study. All subjects had had considerable experience with the experimental procedure before being used in the present study. Following each stimulus, a report 
of "warm," "hot," or "pain" (W, H, or P) was given and an appropriate signal was put on the record by the operator.

In general, there is a sharp increase in the magnitude of the skin resistance change when passing

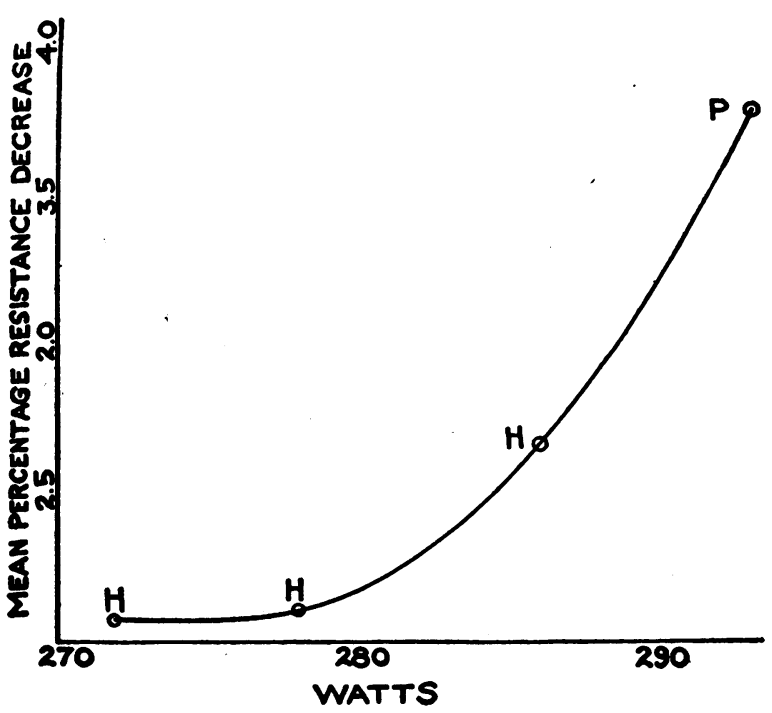

Fig. 1. Change in Skin Resistance Response with Intensity of Stimulus Near Pain Threshold; 124 Determinations in 15 Cases

from an " $H$ " response to a "P." In a few cases, the greatest skin resistance change is observed with the " $\mathrm{H}$ " stimulus which immediately precedes that invoking the response. The course of the response from 124 determinations in 15 individuals is shown in Figure 1. This group included 2 men who showed the greatest resistance change to the
" $\mathrm{H}$ " stimulus. If these had been omitted, the rise with " $P$ " would have been more pronounced.

When the stimulus intensity was maintained very close to the threshold value so that the subjective response was variable, it was found that a larger resistance response was almost invariably associated with the " $P$ " reports.

Both the magnitude and the duration of the skin resistance change increased when the threshold was exceeded (Figure 2). The time from the incidence of the stimulus to the start of the resistance response was independent of the magnitude of the response. In general, the skin resistance returned to its pre-stimulus value in 20 to 30 seconds, which indicates that the 30 -second interval between stimuli cannot be appreciably shortened if independent responses are to be obtained.

When a small dose of morphine ( $8 \mathrm{mgm}$.) was given to a non-addict, an increase in the pain threshold was obtained (Figure 3 ). The change in skin resistance resulting from the " $\mathrm{P}$ " stimuli showed a prompt reduction following the injection and this low value was maintained throughout the period of increased threshold. It has been shown (2) that the post-addict shows a greatly reduced response to the pain-threshold-raising effect of morphine. An $8 \mathrm{mgm}$. dose of morphine produces no significant increase in pain threshold, but is followed by a prompt reduction in the magnitude of the skin resistance response which is quite comparable to that obtained with nonaddicts. In 2 experiments, this reduction persisted for 13 and 14 hours, respectively, at which time
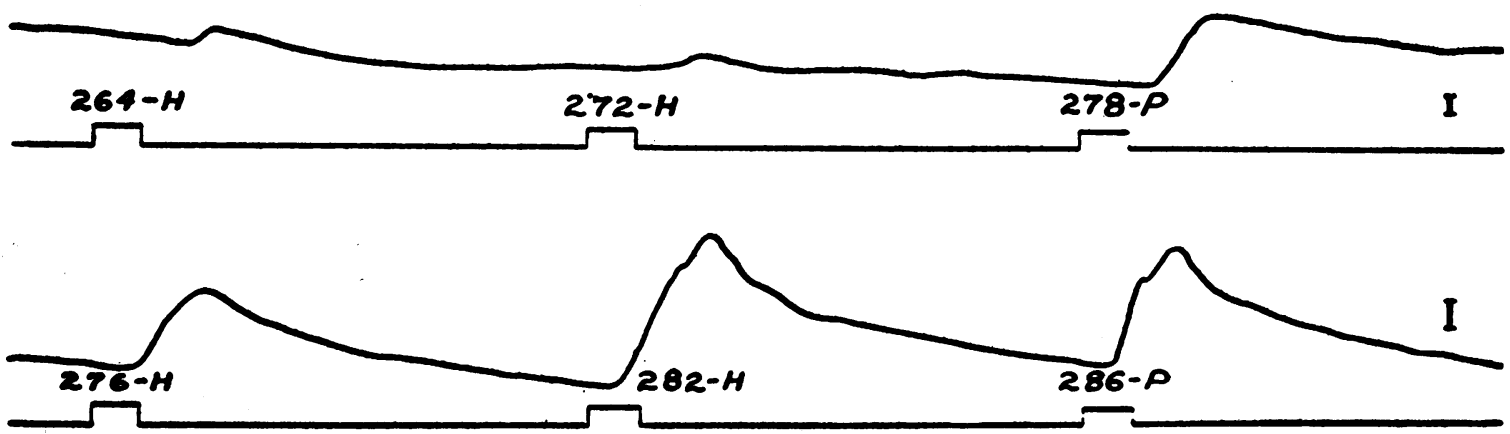

30 SECONDS

1000 OHM CALIBRATNONS

Fig. 2. Changes in Skin Resistance at Threshold

Typical skin resistance responses. The stimulus intensity and the subjective response are recorded at each elevation of the signal line which marks the incidence of the stimulus. 

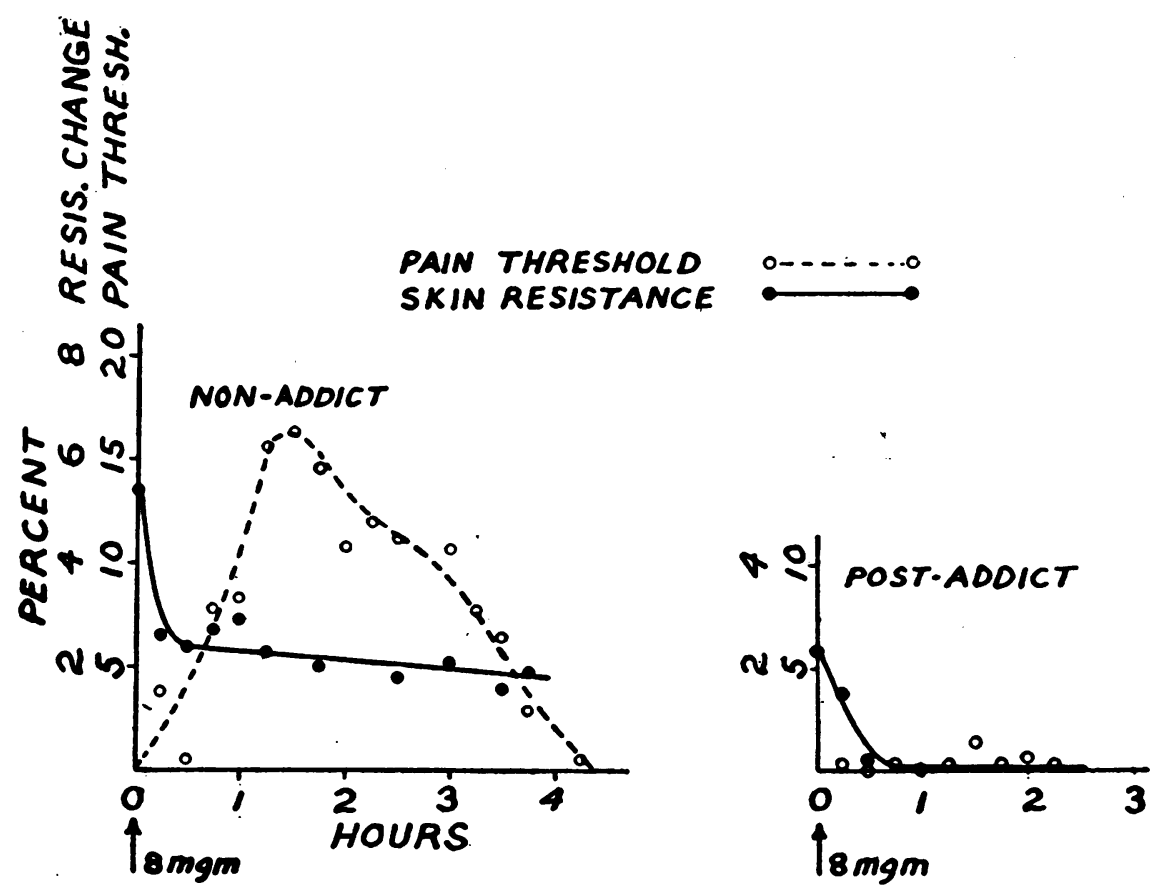

Fig. 3. Effect of Morphine on Pain Theshold and Skin Resistance

Typical changes in the magnitude of the skin resistance response for " $P$ " stimuli following morphine. In both cases, there is a decrease in the skin resistance response but in the post-addict, this is not accompanied by a change in the pain threshold.

the effect started to increase toward the original values. When larger doses (20 to $100 \mathrm{mgm}$.) were given to the post-addict, an increase in pain threshold was obtained and the reduction in the skin resistance change was so marked that no response could be obtained with the recording system at maximum sensitivity.

When an increase in pain threshold was obtained with acetylsalicylic acid instead of with morphine, there was no reduction in the magnitude of the skin resistance response, even though the maximum increases in pain threshold were comparable. Post-addicts showed some reduction in skin response following $100 \mathrm{mgm}$. of codeine, but this reduction was less than that obtained with $20 \mathrm{mgm}$. of morphine, which produces a comparable rise in pain threshold. In the post-addict, the reduction in skin response following $100 \mathrm{mgm}$. of Demerol was at least as great as that produced with $20 \mathrm{mgm}$. of morphine, but the pain-thresholdraising effect of the latter was somewhat greater.

\section{DISCUSSION}

It appears that the skin resistance response cannot be used as an objective measure of the end- point in the determination of pain thresholds, for the response following a " $P$ " report is not invariably greater than with weaker stimuli. In a group, there is a strong tendency for a greater response with a "P" report, which bears out the subjective reports that above threshold, the stimulus has an emotional content considerably greater than stimuli only slightly below threshold. It seems probable that the maximal responses occasionally obtained with the strongest sub-threshold stimuli are due to subjective uncertainty and confusion, producing an autonomic disturbance which is reflected in the increased skin resistance change. When the strength of the stimulus is increased, the uncertainty disappears and the skin response becomes smaller.

Simultaneous measurements of skin resistance and pain threshold are of value, however, in differentiating various aspects of drug action. The action of morphine on that portion of the autonomic system involved in controlling the skin resistance appears to be comparable in post-addicts and normal subjects. However, when the cerebrum becomes involved in the recognition and in- 
terpretation of the pain threshold, there is a distinct difference between the 2 groups.

The fact that morphine produces a reduction in skin response in the post-addict, comparable to that obtained in non-addicts, is of some importance in explaining the clinical relief of pain. It has been shown (2) that the pain-threshold-raising-effect of morphine is greatly reduced in the post-addict, yet clinical relief of pain is obtained with no increase in dosage. The results of the present study indicate that in the post-addict, the mechanisms involved in the skin resistance response are intact, while there is a permanent change in those involved in the recognition of pain threshold. It is interesting that the duration of the effect on skin resistance is about 14 hours, in good agreement with the value given by Himmelsbach (3) for the duration of addiction-satisfying action.

Wolff, Hardy, and Goodell (4) have suggested that the relief of pain by opiates involves at least 2 mechanisms: An increase in pain threshold, and a reduction in the reaction to a recognized pain. They have further shown (5) that the pain threshold reaction is quite independent of this "alarm reaction."

The present studies corroborate these views and suggest that with morphine, the reduction in the alarm reaction is the more important factor. Acetylsalicylic acid appears to relieve pain almost entirely through its pain-threshold-raising action. Codeine exhibits both actions, but the effect on skin resistance is considerably smaller than with morphine. This is in accord with its recognized ability to relieve pain without producing the euphoria and sense of well-being commonly encountered with morphine. The marked effect of Demerol on the skin resistance changes correlates with subjective reports, for this drug produces changes in reaction patterns which are comparable to those of morphine.

\section{CONCLUSIONS}

In general, the skin resistance change produced by the radiant stimulus used in the Hardy-Wolff technique is increased with a subjective report of pain. There are some variations, so this cannot be used as an objective measure of the end-point.

In non-addicts and in post-addicts, there is a reduction in the skin resistance response following a dose of morphine.

There is some reduction following codeine but this is not as pronounced as with morphine.

The reduction following Demerol is at least as great as that observed with morphine.

Following morphine, the reduction. in skin response is maintained considerably beyond the pain threshold effect.

The reduced skin resistance response is probably associated with a reduced pain appreciation, which offers an explanation of the clinical relief of pain in the post-addict.

\section{BIBLIOGRAPHY}

1. Hardy, J. D., Wolff, H. G., and Goodell, H., Studies on pain. A new method for measuring pain threshold: Observations on spatial summation of pain. J. Clin. Invest., 1940, 19, 649.

2. Andrews, H. L., The effect of opiates on the pain threshold in post-addicts. J. Clin. Invest., 1943, 22, 511.

3. Himmelsbach, C. K., The effects of certain chemical changes on the addiction characteristics of drugs of the morphine, codeine series. J. Pharmacol. and Exper. Therap., 1941, 71, 42.

4. Wolff, H. G., Hardy, J. D., and Goodell, H., Studies on pain. Measurements of the effect of morphine, codeine, and other opiates on the pain threshold and an analysis of their relation to the pain experience. J. Clin. Invest., 1940, 19, 659.

5. Wolff, H. G., Hardy, J. D., and Goodell, H., Studies on pain. Measurement of the effect of ethyl alcohol on the pain threshold and on the "alarm" reaction. J. Pharmacol. and Exper. Therap., 1942, 75, 38. 